\title{
Distribuição espacial dos danos de Heilipodus naevulus em plantio de clones de eucalipto
}

\section{Spatial distribution of damage caused by Heillipodus naevulus in a clonal eucalyptus plantation}

\author{
Carlos Augusto Rodrigues Matrangolo ${ }^{1 *}$, Norivaldo dos Anjos ${ }^{2}$, Helio Garcia Leite ${ }^{3}$, \\ Gustavo Eduardo Marcatti ${ }^{3}$
}

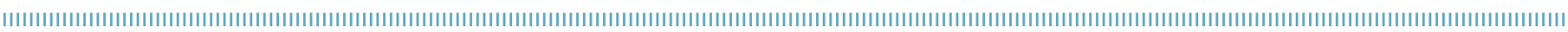

RESUMO: As espécies Heilipodus naevulus (Mannerheim, 1836) foi detectado, em 2008, no município de Barão de Cocais, MG, em plantios comerciais de clones de eucalipto, danificando o ponteiro principal e o lateral. Devido ao seu hábito noturno, o seu tamanho reduzido, coloração acinzentada, e , por se abrigar na área de serapilheira durante o dia, a sua detecçáo é dificultada, sendo a sua presença apenas evidenciada através dos ponteiros tombados. Este trabalho teve como objetivo conhecer a distribuição desta espécie durante o primeiro ano de crescimento clones eucalipto, a fim de fornecer subsídios para a elaboraçáo de um plano de amostragem. $\mathrm{O}$ trabalho foi realizado no município de Barão de Cocais, MG, a partir de outubro/2008 a março/2009. Nos dois talhóes de avaliação foram estratificados em baixada, encosta e topo. Foram distribuídas 18 porçôes, de $10 \times 10$ árvores, sendo nove por talhão, e, três por estrato. Foi adotado como indicador da presença de H.naevulus na planta a presença ponteiro recém danificado. Para o cálculo da distribuição espacial foram empregados a Razão Variância/Média, o Índice de Morisita, o Parâmetro $k$ da distribuição binomial negativa, o Coeficiente de Green, a Distribuição de Poisson e a Lei de potência de Taylor. Ao longo do período de avaliação da presença de $H$. naevulus, os índices calculados apresentaram um padrão agregado de ocorrência dos danos, indicando que a espécie apresenta um padrão agregado de distribuição, mesmo em baixa incidência.

PALAVRAS-CHAVE: insecta; Hylobiinae; Eucalyptus; praga florestal; índice de agregação.
ABSTRACT: The species Heilipodus naevulus (Mannerheim, 1836) was detected, in 2008, in the municipal district Barão de Cocais, MG, in commercial plantings of eucalypt clones, damaging the main and the lateral pointer. Due to its night habit, its reduced size, grayish coloration, and, for sheltering in the litter area during the day, its detection is hindered, being its presence just evidenced through tumbled pointers. This work aimed to know the distribution of this species during the first year of eucalypt clones growth in order to supply subsidies for elaborating of a sampling plan. The work was carried out in the municipal district from Barão de Cocais, MG, from October/2008 to March/2009. The two plots of evaluation were stratified into lowland, slope and top. Were distributed 18 portions, of $10 \times 10$ trees, being nine per plot, and three for stratum. It was adopted as indicator of $H$. naevulus presence in the plant the pointer presence newly damaged. For calculating of the space distribution were used the Variance/Mean Ratio, Morisita Index, Parameter k of the negative binomial distribution, the Green's Coefficient, Poisson's Distribution and the Law of Taylor's potency. Along the evaluation period of the $H$. naevulus presence, the calculated indexes introduced an aggregated pattern of damage occurrence. That Indicates that the species presents a aggregated pattern distribution, even in low incidence.

KEYWORDS: insecta; Hylobiinae; Eucalyptus; forest pest; aggregation index.

'Departamento de Ciências Agrárias; Universidade Estadual de Montes Claros (Unimontes) - Janaúba (MG), Brasil.

${ }^{2}$ Laboratório de Manejo de Pragas Florestais; Universidade Federal de Viçosa (UFV) - Viçosa (MG), Brasil.

${ }^{3}$ Departamento de Engenharia Florestal; UFV - Viçosa (MG), Brasil.

*Autor correspondente: carlos.matrangolo@unimontes.br

Recebido em: 25/07/2012. Aceito em: 10/12/2013. 


\section{INTRODUÇÃO}

Como toda monocultura, as plantaçôes de eucalipto são atacadas por vários grupos de insetos que apresentam potencial para atingir o status de praga e, dentre estes grupos, destacam-se os insetos da ordem Coleoptera. Dentre as espécies, Heilipodus naevulus (Mannerheim, 1836) ainda é pouco conhecida, existindo apenas relatos de sua presença no eucalipto (Silva et al., 1968). Aspectos de sua biologia ainda são pouco conhecidos. Segundo Soria; Dal Conte (2005), os adultos têm hábitos noturnos e causam danos às gemas e aos brotos de videira (Vitis vinifera (L.); durante o dia, permanecem escondidos sob detritos no solo. De acordo com SiLva et al. (1968), os adultos infestam ainda o cajueiro (Anacardium occidentale L.), a espirradeira (Neriun oleander L.), o marmeleiro (Pyrus cydonia L), a nespereira (Eriobotrya japonica Lindl.) e os eucaliptos (Eucalyptus spp.).

Para estabelecer uma amostragem rápida e eficiente, é necessário conhecer o padrão de distribuição populacional das pragas na cultura (FerNANDEs et al., 2003), porque só assim o plano de amostragem se torna confiável (Giles et al., 2000). A distribuição espacial do inseto é utilizada na construção de planos de amostragem para a tomada de decisão sobre o controle das pragas (Boeve; Weiss, 1998), sendo necessário, para isso, que existam dados de contagem de indivíduos. Essas amostragens, de acordo com Young; Young (1998), podem ser utilizadas para inferir sobre a forma de distribuição da população amostrada ou sobre as características dessa distribuição.

Para a descrição das formas de distribuição de uma população são utilizados os índices de agregaçáo e as distribuiçôes de frequências. Os índices de agregação ou dispersão, embora não descrevam matematicamente a distribuição da população estudada (ЕцLiot et al., 1990), fornecem uma ideia aproximada dessa realidade, enquanto diferentes índices fornecem resultados similares (Myers, 1978). O conhecimento das distribuiçôes de probabilidade que descrevem as disposiçôes espaciais de insetos-pragas, que são obtidas a partir dos dados de contagens, é importante para estabelecer critérios adequados de amostragem, análises estatísticas e decisão sobre o controle de pragas agrícolas (Fernandes et al., 2003).

Com a utilização desses modelos de agregação é possível fornecer subsídios para a elaboração de um plano de amostragem para a espécie e, assim, auxiliar com informaçóes para o aprimoramento das técnicas de controle de populações de insetos-praga. Dessa forma, este trabalho tem como objetivo estudar a distribuição espacial dos danos de $H$. naevulus em plantio clonal de eucalipto por meio da determinação dos índices de agregação e dos testes de qui-quadrado de aderência para os principais tipos de distribuiçôes teóricas de frequência.

\section{MATERIAL E MÉTODOS}

O experimento foi conduzido durante o período de outubro/2008 a março/2009, em dois talhôes de plantio comercial de clone de Eucalyptus urophylla $\mathrm{x}$ Eucalyptus sp., denominados, respectivamente, talhão 21/2008 e talhão 29/2008, com área de 31,34 e 48,43 ha. Ambos foram plantados em maio e junho de 2008, estando localizados no município de Barão de Cocais (MG) (1947’56” S, 4327’49” W), e com ocorrência natural de surtos populacionais de $H$. naevulus. O clima da regiáo é classificado como Cwa (tropical de altitude), com chuvas no veráo e inverno seco (classificação de Köppen).

Foram demarcadas nove parcelas de avaliaçáo de 10 x 10 árvores, com total de 900 árvores avaliadas por talhăo. As árvores das parcelas foram identificadas de 1 a 100. Foram feitas avaliaçōes nos meses de outubro e novembro de 2008 e em março de 2009, quando se avaliou a presença de ponteiros atacados nas 18 parcelas.

Os dados da presença de ponteiros recém-atacados foram utilizados para a descrição matemática da dispersão espacial da populaçáo desse inseto na área de estudo. Foram considerados como ataques recentes os ponteiros que ainda apresentavam as folhas túrgidas, cujo ataque ocorreu na noite anterior à avaliaçáo.

Os índices de agregação utilizados foram a razão variância/média, índice de Morisita, parâmetro $k$, coeficiente de Green, distribuiçấo de Poisson e lei de potência de Taylor (Taylor, 1961; Morisita, 1962; Boeve; Weiss, 1998; YounG; Young, 1998; Fernandes et al., 2003; CosTA et al., 2006; MARUYAMA et al., 2006).

\section{RESULTADOS E DISCUSSÃO}

$\mathrm{O}$ uso da presença de ponteiros recém-atacados para calcular o padrão de atividade daninha de $H$. naevulus em uma área de plantio clonal de eucalipto foi utilizado devido à facilidade de localizá-los nas árvores e brotações, e também pelo comportamento noturno da espécie. $\mathrm{O}$ inseto é relativamente pequeno e só sai para se alimentar após o crepúsculo, quando provoca dano no ponteiro do eucalipto. $\mathrm{O}$ ataque foi considerado recente quando o local atacado ainda se apresentava túrgido, sem sinais de dessecamento do ponteiro tombado. Os resultados dos índices de agregação calculados são apresentados na Tabela 1 e nas Figuras 2 a 6.

Os resultados obtidos na razão variância/média (I) durante o período de avaliaçáo da ocorrência de ataques no plantio foram maiores que um, demonstrando que a distribuiçấo de plantas atacadas nas parcelas avaliadas apresentou distribuição agregada. Os maiores valores observados nos meses de outubro 
e novembro refletem o maior número de observaçóes de ataque nas áreas avaliadas, uma vez que quanto maior o valor de $I$, mais agregada a espécie.

Os valores do índice de Morisita $\left(I_{\delta}\right)$ calculados no período de avaliação foram superiores a um, confirmando que a espécie apresenta padrão de distribuição agregada. Nesse caso, o maior valor do índice, observado no mês de março, indica que mesmo em baixa população a espécie apresenta padrão agregado. Os valores de $X^{2} e I_{\partial}$ confirmam o padrấo de agregação de ataque da espécie em todos os meses de avaliaçáo. Como ocorre em $I$, o maior valor de $I_{\delta}$ indica maior agregação da espécie.

$\mathrm{O}$ parâmetro $k$ da distribuição binomial negativa variou de 0,1467 a 2,6152, ficando entre 0 e 8 , indicando agregação da espécie. No mês de março, quando obteve-se o índice mais baixo $(0,1467)$, a agregaçáo foi maior, pois a máxima aproximação entre os indivíduos acontece quando o valor tende a zero. Nos demais meses, os índices também ficaram baixos, indicando maior agregação entre os indivíduos.

Por meio da análise do coeficiente de dispersão de Green $(C x)$, para todos os meses avaliados foram registrados valores maiores que zero, indicando distribuição agregada. Os valores mostram baixa intensidade de agregação, visto que quanto mais próximo de zero, menos agregada é a distribuiçấo. No caso do mês de março, apesar da menor ocorrência de $H$. naevulus, foi verificado um valor maior de $C x$, o que mostra maior proximidade dos indivíduos.

Para estudar a distribuição da frequência de ataques da praga, os dados foram ajustados à distribuição de Poisson e, em todas as amostragens dos talhóes 21/2008 e 29/2008, os valores de $\chi^{2}$ foram significativos ( $\mathrm{p} \leq 0,05$ ), o que revela que a distribuição não é aleatória. Como as variâncias foram superiores às médias, testou-se em seguida o ajuste à distribuição binomial negativa.

As estimativas dos parâmetros da Lei de Potência de Taylor foram obtidas aplicando-se uma linha de tendência de potência para a variância e a média dos dados das amostragens (Fig. 1).
O valor calculado do parâmetro $b$ da Lei de Potência de Taylor para a ocorrência de ataque de $H$. naevulus foi de 1,3433 , e o valor do teste $t$ para estimativa dos parâmetros, para ajuste do modelo e para a hipótese $H O: b=1$ versus $H 1$ foram significativos $(\mathrm{p} \leq 0,05)$. O valor do parâmetro $b$ indicou um padrão de agregação da espécie durante todos os períodos de avaliaçáo (Tabela 2).

Em todos os índices calculados evidenciou-se um padrão agregado. Esse padrão foi observado em diferentes espécies de pragas (Fernandes et al., 2003; Costa et al., 2006; Maruyama et al., 2006) e, aqui, foi constatado em todos os meses de avaliaçấo do trabalho. Isso sugere a possibilidade de que a espécie de inseto, mesmo em baixa populaçáo, apresente um padrão de distribuição espacial de ataque do tipo agregado (Figs. 5 e 6 ).

Quando se analisa o padrão de distribuição de ataque da espécie na área de plantio (Figs. 2 a 6) e a percentagem de árvores atacadas por talhão (Tabela 3), verifica-se que o talhão 21/2008 apresentou, nos meses de outubro e novembro, maior porcentagem de árvores atacadas em relação ao talhão 29/2008 (Figs. 2 e 3). O padrão de distribuição de

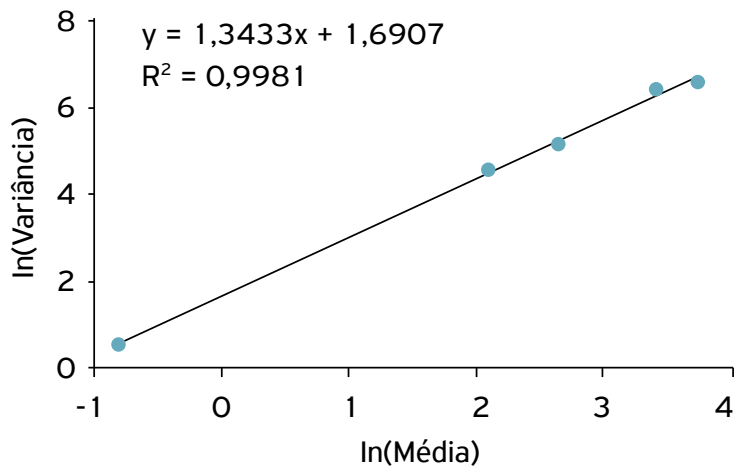

Figura 1. Linearização da Lei de Potência de Taylor $\left(s^{2}=a+\hat{m} \cdot b\right)$ para estudo de agregação de ataque de Heilipodus naevulus (Mannerheim, 1836) utilizando-se $\ln \mathrm{S}^{2}=\ln \mathrm{a}+\mathrm{b} \ln \hat{\mathrm{m}}$, Antilog $\mathrm{a}=5,4233$. Barão de Cocais (MG), 2009.

Tabela 1. Estimativas do padrão de distribuição espacial de Heilipodus naevulus (Mannerheim, 1836) através dos índices de agregação em plantio de clones de eucalipto. Barão de Cocais (MG), 2009.

\begin{tabular}{|c|c|c|c|c|c|}
\hline \multirow{2}{*}{ Índices } & \multicolumn{5}{|c|}{ Datas de avaliação } \\
\hline & Outubro(1) & Novembro(1) & Janeiro(2) & Fevereiro(2) $^{(2)}$ & Março(2) \\
\hline$\hat{\mathrm{m}}$ & 30,5 & 42,2778 & 0,1956 & 0,1411 & 0,0667 \\
\hline $\mathrm{S}^{2}$ & 618,2727 & 725,7418 & 0,2130 & 0,1813 & 0,1245 \\
\hline I & 20,2712 & 17,1660 & 12,7815 & 11,7615 & 4,0294 \\
\hline$l_{\partial}$ & 1,5808 & 1,3616 & 1,7980 & 2,2617 & 8,3571 \\
\hline$X^{2} \mid e I_{\partial}$ & $222,9836^{*}$ & $291,8226^{*}$ & $217,2857^{*}$ & $199,9452^{*}$ & $68,5^{*}$ \\
\hline Valor de $\mathrm{p} \mathrm{I} \mathrm{e} \mathrm{I}$ & 0 & 0 & 0 & 0 & 3,90E-08 \\
\hline K & 1,5827 & 2,6152 & 1,1883 & 0,7537 & 0,1467 \\
\hline$C x$ & 0,0528 & 0,0213 & 0,0469 & 0,0742 & 0,4328 \\
\hline
\end{tabular}

(1): 2008; (2): 2009; $\hat{m}$ média amostral; $S^{2}$ :variância amostral; I: razão variância/média; $I_{\delta}$ : índice de Morisita; $X^{2}$ Ie $I_{\delta}$ : teste de afastamento da aleatoriedade; valor de p / $l_{\beth}$ : nível de probabilidade do teste qui-quadrado; $k$ : parâmetro $k$ : $C x$ : coeficiente de Green; *: significativo a $5 \%$ de probabilidade. 
Tabela 2. Estimativas dos parâmetros da Lei de Potência de Taylor e estatísticas da análise de regressão para estudo de agregação de ataque de Heilipodus naevulus (Mannerheim, 1836). Barão de Cocais (MG), 2009.

\begin{tabular}{lccccc} 
Parâmetros & Estimativa & Teste $t^{(1)}$ & $\mathbf{R}^{2}$ & Teste $\mathbf{F}^{(2)}$ & Teste $t^{(3)}$ \\
\hline A & 1,6907 & $18,3^{* *}$ & & & \\
\hline B & 1,3433 & $39,92^{* *}$ & 0,9981 & $1594^{* *}$ & $10,2024^{* *}$ \\
\hline
\end{tabular}

(1): teste $t$ para estimativa dos parâmetros; ${ }^{(2)}$ : teste $F$ para ajus te do modelo; ${ }^{(3)}$ : teste $t$ para a hipótese $H O: b=1$ vs. $H 1: b \neq 1$;

**: Significativo $(p \leq 0,01)$.

Tabela 3. Porcentagem de árvores atacadas nos meses de outubro e novembro de 2008 , e nos talhões $21 / 2008$ e $29 / 2008$ nos respectivos meses. Barão de Cocais (MG).

\begin{tabular}{|c|c|c|c|c|c|c|c|c|c|}
\hline & $\mathbf{N}$ & 0 & 1 & Morta & Talhão № & $\mathbf{N}$ & 0 & 1 & Morta \\
\hline \multirow{2}{*}{ Outubro } & \multirow{2}{*}{1200} & \multirow{2}{*}{67,5} & \multirow{2}{*}{30,50} & \multirow{2}{*}{2,00} & $21 / 2008$ & 600 & 56,50 & 42,83 & 0,67 \\
\hline & & & & & 29/2008 & 600 & 78,50 & 18,17 & 3,33 \\
\hline \multirow{2}{*}{ Novembro } & \multirow{2}{*}{1800} & \multirow{2}{*}{55,94} & \multirow{2}{*}{42,28} & \multirow{2}{*}{1,78} & $21 / 2008$ & 900 & 41,00 & 58,11 & 0,89 \\
\hline & & & & & $29 / 2008$ & 900 & 70,89 & 26,44 & 2,67 \\
\hline
\end{tabular}

N: número de árvores avaliadas; 0 : árvores não atacadas; 1 : árvores atacadas; Morta: árvores eliminadas devido à falha no plantio, quebrada pelo vento ou devido ao ataque de Heilipodus naevulus (Mannerheim, 1836).
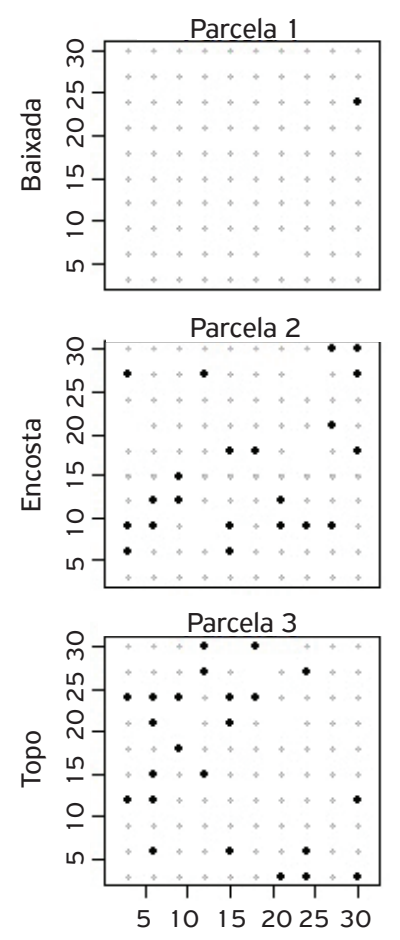
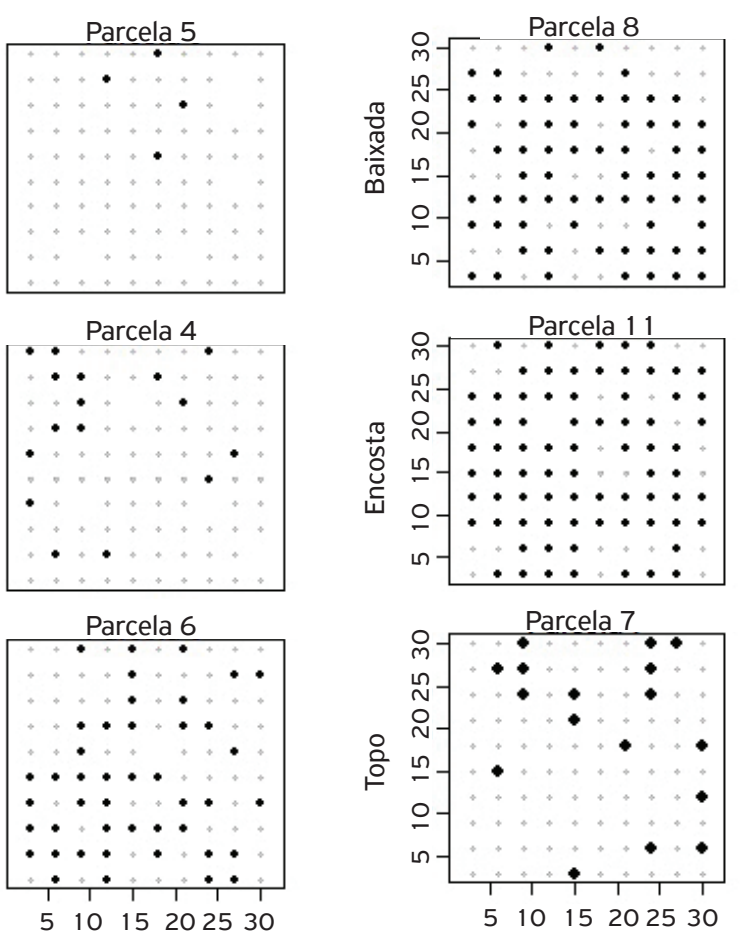
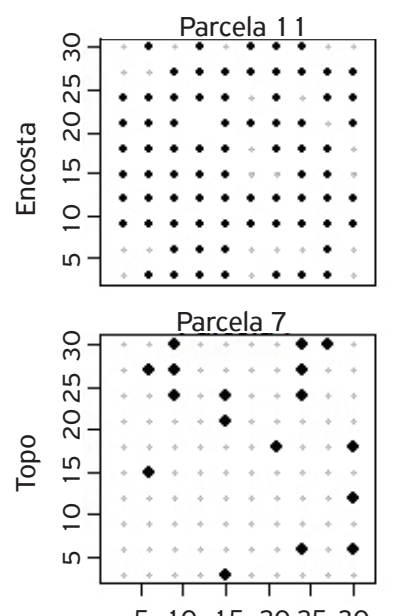

51015202530
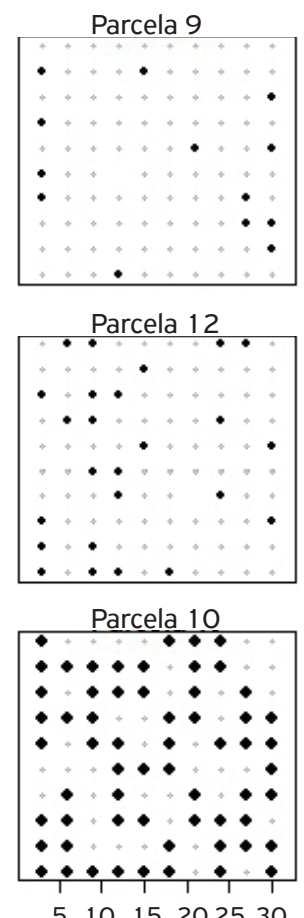

•: árvores não atacadas; •: árvores atacadas; espaço vazio: árvores mortas. Valores dos eixos x e y correspondem às distâncias entres as árvores, com total de $30 \mathrm{~m}$.

Figura 2. Distribuição do ataque de Heilipodus naevulus (Mannerheim, 1836) em áreas de plantio de clones de eucalipto, plantados sob arranjo espacial de 3 × 3 m, no mês de outubro/2008, nos talhões 21 ( 7 - 12) e 29 (1 - 6), subdivididos em baixada, encosta e topo. Barão de Cocais (MG).

ataque observado nos talhôes avaliados, mesmo em baixa incidência, é um indicativo de que a espécie deve apresentar um padrão agregado.

Devido à inexistência de estudos sobre a distribuição espacial de danos causados por besouros desfolhadores em plantio comercial de eucalipto no Brasil, náo foi possível comparar o padrão registrado com o de outros coleópteros. A partir do conhecimento do padrão de ataque apresentado pela espécie, é possível definir estratégias para o manejo integrado da mesma. Porém, falta determinar o que a leva a apresentar padrão de ataque do tipo agregado. Tal fato pode estar associado ao uso de certos 

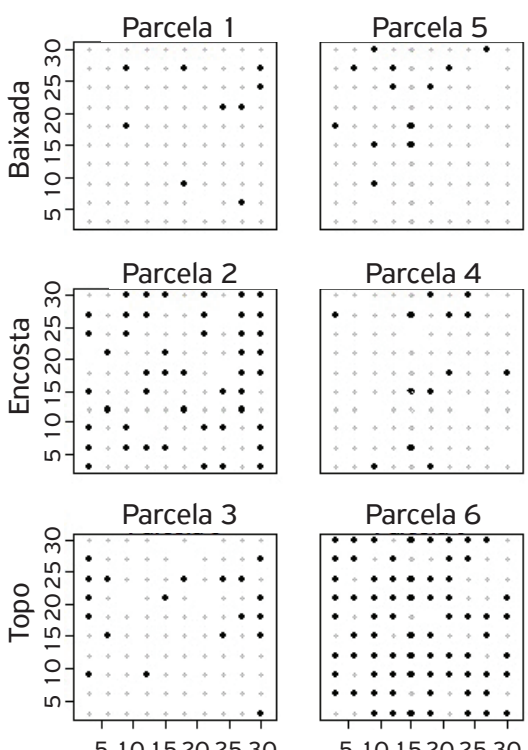

51015202530

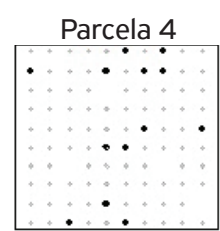

Parcela 6

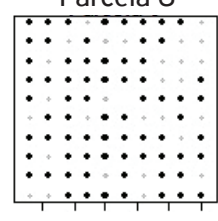

51015202530
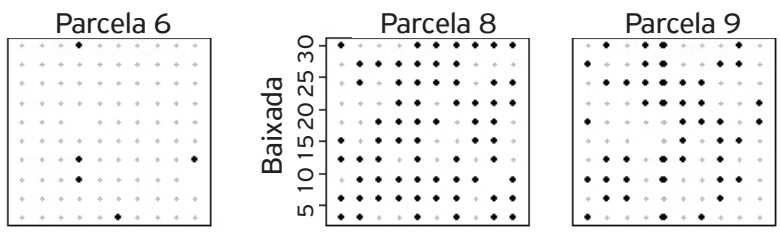

Parcela 13

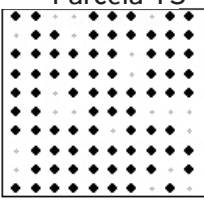

Parcela 17

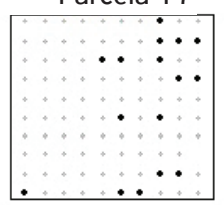

Parcela 11

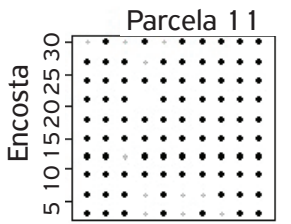

Parcela 12

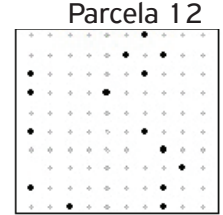

Parcela 7
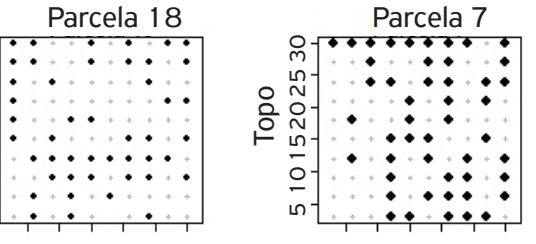

51015202530
Parcela 15

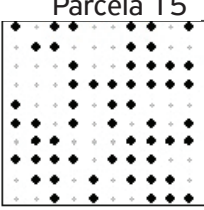

Parcela 14

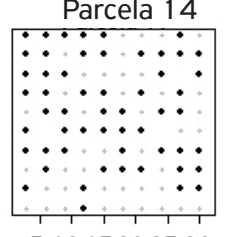

-: árvores não atacadas; •: árvores atacadas; espaço vazio: árvores mortas. Valores dos eixos x e y correspondem às distâncias entres as árvores, com total de $30 \mathrm{~m}$.

Figura 3. Distribuição do ataque de Heilipodus naevulus (Mannerheim, 1836) em áreas de plantio de clones de eucalipto, arranjo sob espacial de 3 x 3 m, no mês de novembro /2008, nos talhões 21 (7 - 12, 16 - 18) e 29 ( 1 - 6, 13 - 15), subdivididos em baixada, encosta e topo. Barão de Cocais (MG).
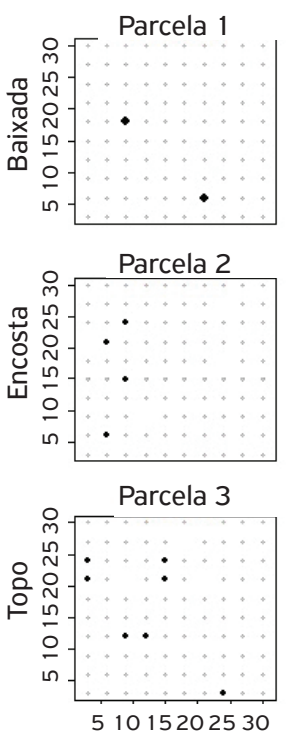

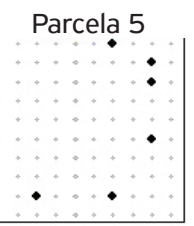

Parcela 4

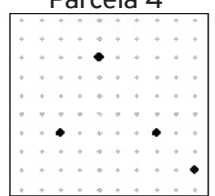

Parcela 6

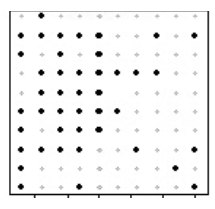

51015202530

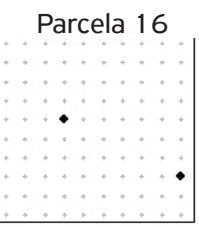

Parcela 17

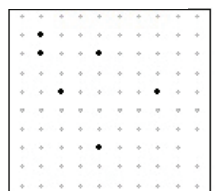

Parcela 18

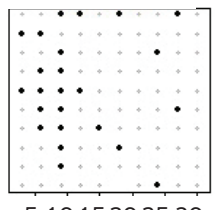

51015202530
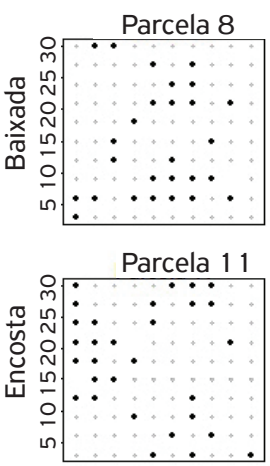

Parcela 7

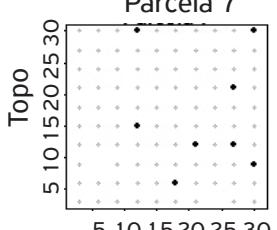

51015202530

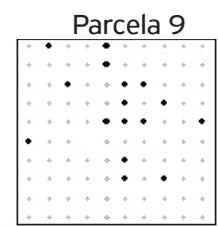

Parcela 12

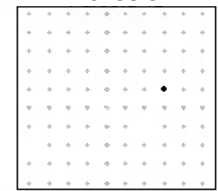

Parcela 10

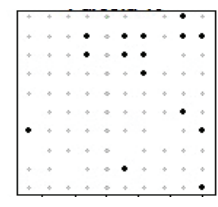

51015202530

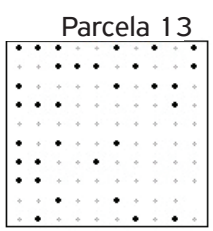

Parcela 15

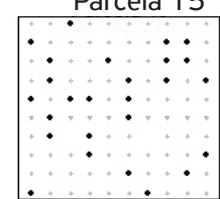

Parcela 14

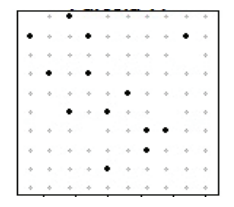

51015202530

•: árvores não atacadas; •: árvores atacadas; espaço vazio: árvores mortas. Valores dos eixos x e y correspondem às distâncias entres as árvores, com total de $30 \mathrm{~m}$.

Figura 4. Distribuição do ataque de Heilipodus naevulus (Mannerheim, 1836) (metro) em áreas de plantio de clones de eucalipto, sob arranjo espacial de 3 x 3 m, no mês de janeiro/2009, nos talhões 21 ( 7 - 12, 16 - 18) e 29 ( 1 - 6, 13 - 15), subdivididos em baixada, encosta e topo. Barão de Cocais (MG).

feromônios. Ambrogi et al. (2009), revisando o conhecimento sobre feromônios de agregação em Curculionidae, ressaltaram que os produzidos pelas fêmeas incluem feromônios sexuais de longa distância, que atraem principalmente machos; curta distância ou feromônio de contato, que permite reconhecer coespecíficos; e feromônio de oviposição, que algumas fêmeas usam para marcar os hospedeiros. Como salientaram os autores, o uso de feromônios é uma técnica bastante promissora para o manejo integrado de pragas.

Para melhor determinar estratégias para o manejo integrado de $H$. naevulus, estudos complementares sobre a 

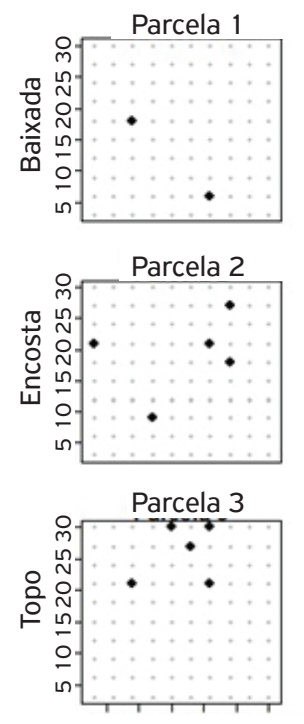

51015202530
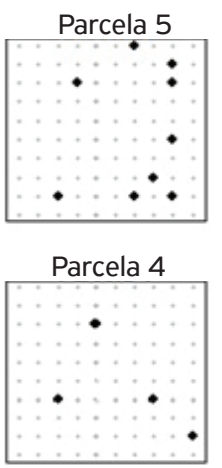

Parcela 6

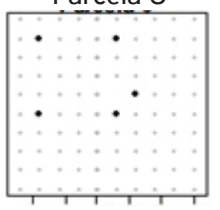

51015202530
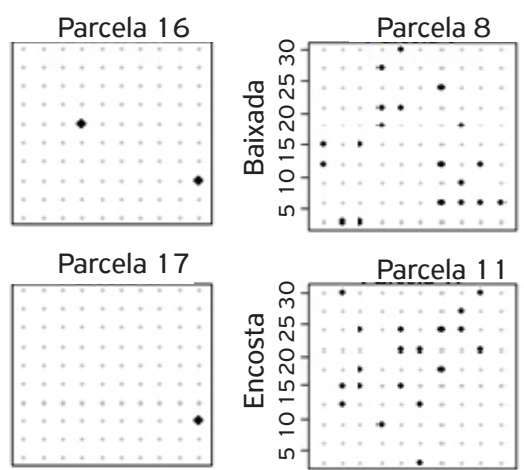

Parcela 18

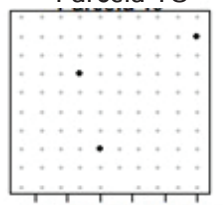

51015202530
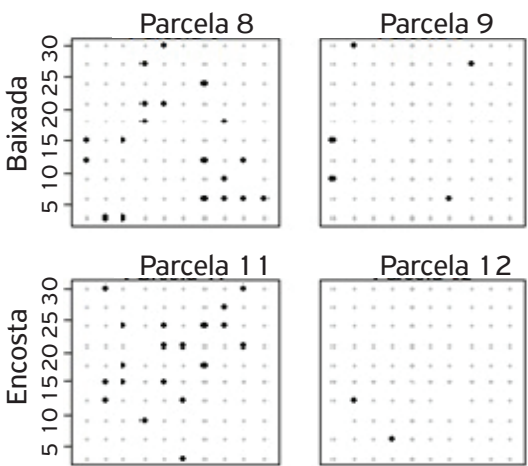

Parcela 10

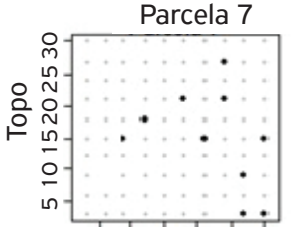

51015202530

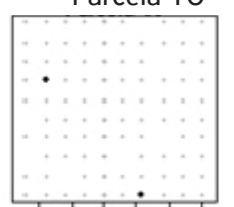

51015202530

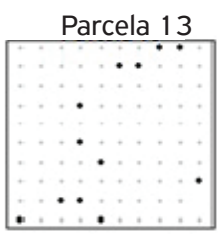

Parcela 15

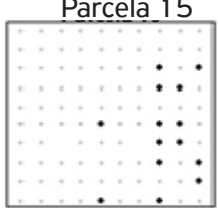

Parcela 14

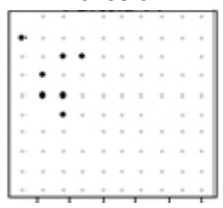

51015202530

•: árvores não atacadas; •: árvores atacadas; espaço vazio: árvores mortas. Valores dos eixos x e y correspondem às distâncias entres as árvores, com total de $30 \mathrm{~m}$.

Figura 5. Distribuição do ataque de Heilipodus naevulus (Mannerheim, 1836) em áreas de plantio de clones de eucalipto, plantados sob arranjo espacial de $3 \times 3$ m, no mês de fevereiro/2009, nos talhões $21(7-12,16$ - 18) e 29 (1 - 6, 13 - 15), subdivididos em baixada, encosta e topo. Barão de Cocais (MG).
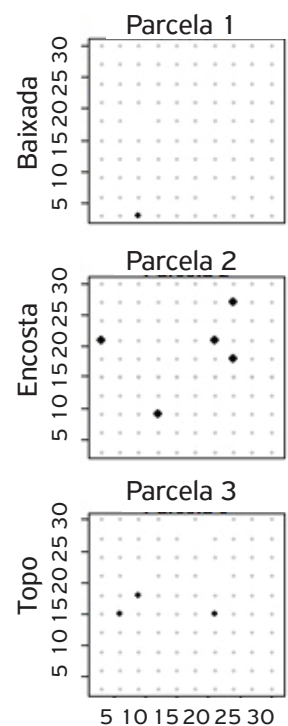

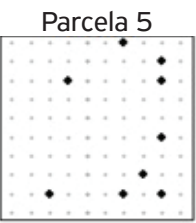

Parcela 4

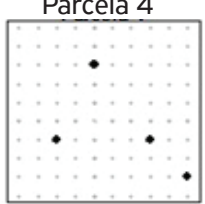

Parcela 6

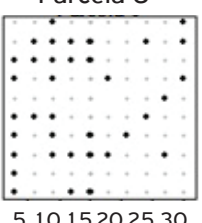

51015202530

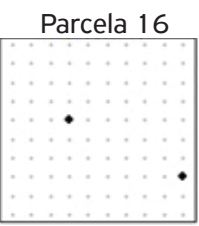

Parcela 17

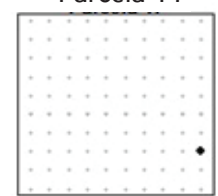

Parcela 18

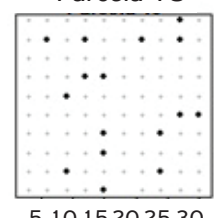

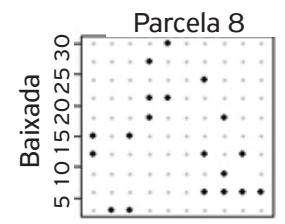
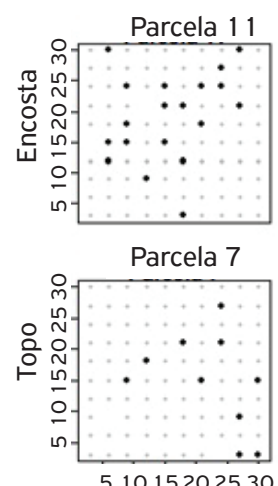
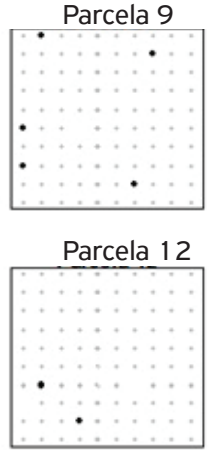

Parcela 10

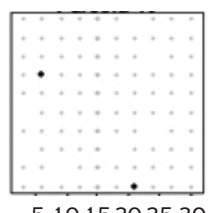

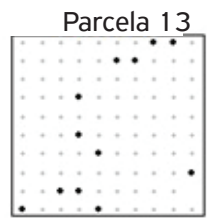

Parcela 15

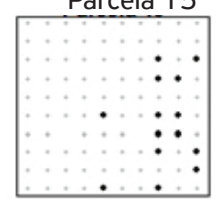

Parcela 14

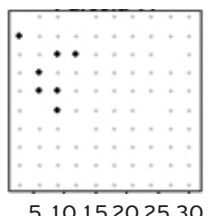

•: árvores não atacadas; •: árvores atacadas; espaço vazio: árvores mortas. Valores dos eixos x e y correspondem às distâncias entres as árvores, com total de $30 \mathrm{~m}$.

Figura 6. Distribuição do ataque de Heilipodus naevulus (Mannerheim, 1836) em áreas de plantio de clones de eucalipto, sob arranjo espacial de 3 x 3 m, no mês de março/2009, nos talhões 21 ( 7 - 12, 16 - 18) e 29 ( 1 - 6, 13 - 15), subdivididos em baixada, encosta e topo. Barão de Cocais (MG).

distribuiçấo espacial dos adultos e de sua mobilidade deveriam ser conduzidos.

Os resultados obtidos pelos índices calculados indicam que existe, mesmo quando se constata uma baixa incidência da população, distribuição espacial do tipo agregado para os estragos causados pelos adultos de Heilipodus naevulus nos talhóes amostrados.

\section{AGRADECIMENTO}

À Celulose Nipo-Brasileira S/A (CENIBRA), pela oportunidade de trabalhar em suas áreas de plantio para a coleta dos dados do trabalho. À Fundação de Amparo à Pesquisa do Estado de Minas Gerais (FAPEMIG), pela concessão de bolsa de doutoramento, permitindo a realização do trabalho. 


\section{REFERÊNCIAS}

AMBROGI, B.G.; VIDAL, D.M.; ZARBIN, P.H.G. Feromônios de agregação em Curculionidae (Insecta: Coleoptera) e sua implicação taxonômica. Química Nova, v.32, n.8, p.2151-2158, 2009.

BOEVE, P.J.; WEISS, M. Spatial distribution and sampling plans with fixed levels of precision for cereal aphids (Homoptera:Aphididae) infesting spring wheat. Canadian Entomologist, v.130, p.6777, 1998.

COSTA, M.G.; BARBOSA, J.C.; YAMAMOTO, P.T. Distribuição de Probabilidade de Ocorrência de Orthezia praelonga Douglas (Hemiptera: Sternorrhyncha: Ortheziidae) na Cultura de Citros. Neotropical Entomology, v.35, n.3, p.395-401, 2006.

ELLIOT, N.C.; KIECKHEFER, R.W.; WALGENBACH, D.D. Binomial sequential sampling methods for cereal aphids in small grains. Journal of Economic Entomology, v.83, p.1381-1387, 1990.

FERNANDES, M.G.; BUSOLI, A.C., BARBOSA, J.C. Distribuição Espacial de Alabama argillacea (Hübner) (Lepidoptera: Noctuidae) em Algodoeiro. Neotropical Entomology, v.32, n. 1, p.107-115, 2003.

GILES, K.L.; ROYER, T.A.; ELLIOTT, N.C.; KINDLER, S.D. Development and validation of a binomial sequential sampling plan for the greengug (Homoptera: Aphididae) infesting winter wheat in the southern plains. Journal of Ecomomic Entomology, v.93, p.1522-1530, 2000.

MARUYAMA, W.I.; BARBOSA, J.C.; TOSCANO, L.C. Distribuição Espacial de Oncometopia facialis (Signoret) (Hemiptera: Cicadellidae) em Pomar Cítrico. Neotropical Entomology, v.35, n. 1, p.93-100, 2006.

MORISITA, M. I I- Index, a measure of dispersion of individuals. Research Population Ecology, v.IV, p. 1-7, 1962.

MYERS, J.H. Selecting a measure of dispersion. Environment Entomolology, v.7, p.619-621, 1978.

SILVA, A.G.A.; GONÇALVES, C.R.; GALVÃO, D.M.; GONÇALVES, A.J.L.; GOMES, J.; SILVA, M.N. \& SIMONI, L. Quarto Catálogo dos Insetos que vivem nas plantas do Brasil. Rio de Janeiro: Ministério da Agricultura. 1968.

SORIA, S.J.; DAL CONTE, A.F. Bioecologia e controle de pragas da videira. Circular Técnica 63, Embrapa Uva e Vinho. 2005. 20p.

TAYLOR, L.R. Aggregation, variance and the mean. Nature, v.189, p.732-735, 1961

YOUNG, L.J.; YOUNG, J.H. Statistical ecology: a population perspective. Boston, Kluwer Academic Publishers, 1998. 565p. 